\title{
DENTAL TRAUMA: ASSESSMENT OF UNDERGRADUATE DENTAL STUDENTS: THEORETICAL KNOWLEDGE AND PRACTICAL USE OF SMARTPHONE APPLICATION 'INJURED TOOTH'
}

\author{
Adel Abdel Azeem ElBardissy*
}

\begin{abstract}
Background: Few studies investigated the teaching of Dental Trauma at undergraduate levels, and explored the effectiveness of some teaching interventions on students. The last decade has seen an increasing use of advanced technology within the arena of dental educational intervention. The trend certainly anticipates the overall improvement towards reaching a precise diagnosis and appropriate management of traumatic dental injuries. Some Smartphone applications are examples of this educational technology.
\end{abstract}

Aim: It firstly aims to evaluate the knowledge of final year undergraduate students about dental trauma. Secondly, the study further assesses the ability of a selected group of the students to diagnose and propose treatment plan/s for different dental traumatic injuries using a Smartphone Application 'Injured Tooth'.

Design: Dental undergraduate final year students were invited to complete a questionnaire on dental trauma after finishing all the lectures and relevant Case Studies' Sessions. The same questionnaire was used to test changes in the knowledge level of sixty students selected to use Injured Tooth application.

Results: 274 students agreed to participate in the research. The mean percentage of correct answers for all the questions was $77.8 \%$ (SD 17.3). The mean values for the total score were 8.4 (SD 1.4) with a median of 8 and a range of $5-11$. The mean percentage of correct answers of the selected students was 68.97 (SD 20.2). The mean percentage of correct answers of the students using the application was $95.8(\mathrm{SD} 7.0)$.

Conclusions: Knowledge of the students regarding traumatic dental injuries is efficient in some areas while considered low in other parts. The use of the application was effective in providing accessible knowledge to the students to guide them in proposing the possible management protocols for the different traumatic dental injuries presented in this study.

KEYWORDS: Dental trauma - students' knowledge - smartphone applications.

*Associate Professor of Pediatric Dentistry, Cairo University. 


\section{INTRODUCTION}

Traumatic dental injuries are considered the second most frequent dental problem, with dental caries being the first, with a permanent dentition prevalence of $15.2 \%$ and a primary dentition prevalence of $22.7 \%$ worldwide ${ }^{(1)}$.

In Egypt, the prevalence of traumatic dental injuries represents $10 \%$ for primary dentition ${ }^{(2)}$ and $14.6 \%$ in permanent dentition ${ }^{(3)}$. This relatively high prevalence motivates the dental professionals to use all the available diagnostic aids and technology to reach a correct diagnosis and a suitable treatment plane for these injuries.

In fact, it is a complex procedure to establish an ideal diagnosis and treatment for traumatic dental injuries. There are nine fracture types and six luxation types, which might occur solely or combined, affecting either the primary or the permanent dentitions or both. Each type of trauma has a specific treatment plan and a predicted prognosis. With such a complexity in diagnosis and treatment, it is obvious that well-experienced practitioners may have problems in selecting the proper treatment plan in certain trauma types ${ }^{(4)}$.

The primary management provided at the time of injury is a crucial factor in the long-term success ${ }^{4}$. The cornerstone is reaching a correct diagnosis in order to provide the appropriate treatment. The accuracy of diagnosis should be based both on the clinical as well as radiographic findings, history, and the experience of the treating clinician ${ }^{(5)}$.

With inadequate knowledge, the dentist cannot provide the best possible treatment for the traumatically-injured patient. The existing dental curriculum for undergraduate students in Cairo University, Egypt, stipulates that upon the completion of dental traumatic injuries educational theoretical lectures and sessions; the students should be in a better position to both diagnose and identify treatment of traumatic dental injuries, both in primary and permanent teeth, and to predict the prognosis of such injuries.
The available literature reveals that few studies have been conducted to investigate students' knowledge or the efficiency of the applied teaching interventions on their knowledge. These studies addressed various interventions, such as: the effect of pediatric dental education of dental traumatology (6); and attending an extra single condensed refreshing lecture on dental trauma ${ }^{(7,8)}$, another looked at different factors to test their influence on dental students' level of educational knowledge and management of traumatic injuries. These factors included: gender, marital status, and the university where the subject students graduated at, and traumatic dental injury management experience ${ }^{(9)}$.

The use of advanced technology in the education process, as an educational intervention, has increased in the last decade, aiming at improving the knowledge regarding the correct diagnosis and at managing of traumatic dental injuries successfully (10). Smartphones contributed to these technologies, launching several Applications to help clinicians in their diagnosis and planning for treatment of traumatic dental injuries ${ }^{(11)}$.Dentists and other allied health care staff around the world often use mobile Applications as part of their professional practice (12).

In the year 2015, a study has revealed the availability of eight Applications for traumatic dental injuries; with only one Application, namely; 'AcciDent', was designated for professional dental use ${ }^{(13)}$. Recently; the International Association of Dental Traumatology launched a free Application, named: 'ToothSOS', designated to help members from the public in handling emergency dental traumatic injuries; in other words: for domestic use. It is available in eight languages ${ }^{(14)}$. The Arabic language is not included, however.

In the year 2016, a new Application for professional use named 'Injured Tooth' was launched to assist in diagnosing traumatic injuries to the teeth and to supporting structures. Additionally, it describes the management protocols for each 
trauma type both for primary and permanent dentitions, as per the Guidelines of the International Association of Dental Traumatology. To make a diagnosis, the users initially select a type of injury, and then answer a series of questions. Based on those answers, the Application provides a diagnosis ${ }^{(15)}$.

A study published in 2018 studied the sum of 201 cases, and concluded that the 'Injured Tooth' Application provided the correct diagnosis for different traumatic injuries in $98 \%$ of the cases ${ }^{(16)}$.

There is limited evidence that this Application, i.e. 'Injured Tooth', is in use as an undergraduate educational intervention, and in regard to its effectiveness amongst the final years' students, Faculty of Dentistry, Cairo University, Egypt.

This Study aims to: firstly; to evaluate the knowledge possessed by final year' students, in the area of dental trauma management. Typically, at the Faculty of Dentistry, Cairo University, the final year's students do attend lectures and relevant Case Studies' Sessions, according to the established pediatric dentistry curriculum. Secondly, the Study aims to examine the change in the ability of the final year' students to diagnose and propose treatment plan/s for different dental traumatic injuries using the Smartphone Application 'Injured Tooth'. The second phase of the Study is carried out on a selected sample from those students.

\section{MATERIALS AND METHODS}

This study was approved by the Research Ethics Committee, Faculty of Dentistry,Cairo University. The Study comprises two phases. The first phase (Phase A) is to evaluate the final year's students for their knowledge in management of dental trauma after attending the lectures and the Case Studies' discussions on dental traumatic injuries, according to the applicable pediatric dental curriculum, the faculty of Dentistry, Cairo University. Out of 375 final years' students, 274 agreed to participate in the research. The second phase (Phase B) is to examine the ability of a selected sample from these students, in diagnosing and proposing the treatment plan/s for different dental traumatic injuries through using the Application: 'Injured Tooth' .

A questionnaire is used to accomplish Phase A: the knowledge of the final years' students (Fig. 1). The Questionnaire includes two Sections: the first Section is to gather the personal details; explicitly their names, and preferred contact numbers (in order to call the selected students for Phase B of the Study). The first Section also includes questions about gender, rank of the preceding year, and whether the student has had a previous clinical experience regarding management of dental trauma. The answers of these questions are used as predictor variables in the analysis of the results. The second Section of the Questionnaire includes questions to evaluate the ability of each student to diagnose and select the appropriate treatment for three given dental traumatic injuries cases: a case of complicated crown fracture, secondly a case of an Intrusive luxation, and lastly a case of an avulsion. Concurrently, cases were presented as a slideshow, with all the required information detailed in the Questionnaire (Figs. 2, 3,\& 4).

Students who attended the last session of the Case Studies over a week were asked to complete the Questionnaire, during the actual session. They were allowed 15 minutes to complete the Questionnaire.

Students who had wrong answers in the Questionnaire were clustered into three categories: those who got wrong diagnoses, those who got wrong answers, and those whose answers were considered deleterious on treatment and prognosis. The researcher selected 60 students from those three categories, and asked each to proceed to Phase B of the Study.

At the commencement of Phase B, the faculty member of the research presented a demonstration session to the selected students on how to operate and navigate the Application's different features. The Researcher installed the Application 'Injured 
Tooth' on 5Smartphones held by Department of Pediatric Dentistry. Subsequently, the identified students were requested to re-answer the same Questionnaire, using the Application, and were given fifteen minutes to accomplish the task.

\section{Description: 'Injured Tooth’ Application}

The 'Injured Tooth' Application is produced by Ignobilis Terrain LLP, and endeavors to provide the diagnosis of traumatic injuries to the teeth and supporting structures. Moreover, it describes all probable management protocols for each trauma type; for both primary and permanent dentitions, according to the Guidelines of the International

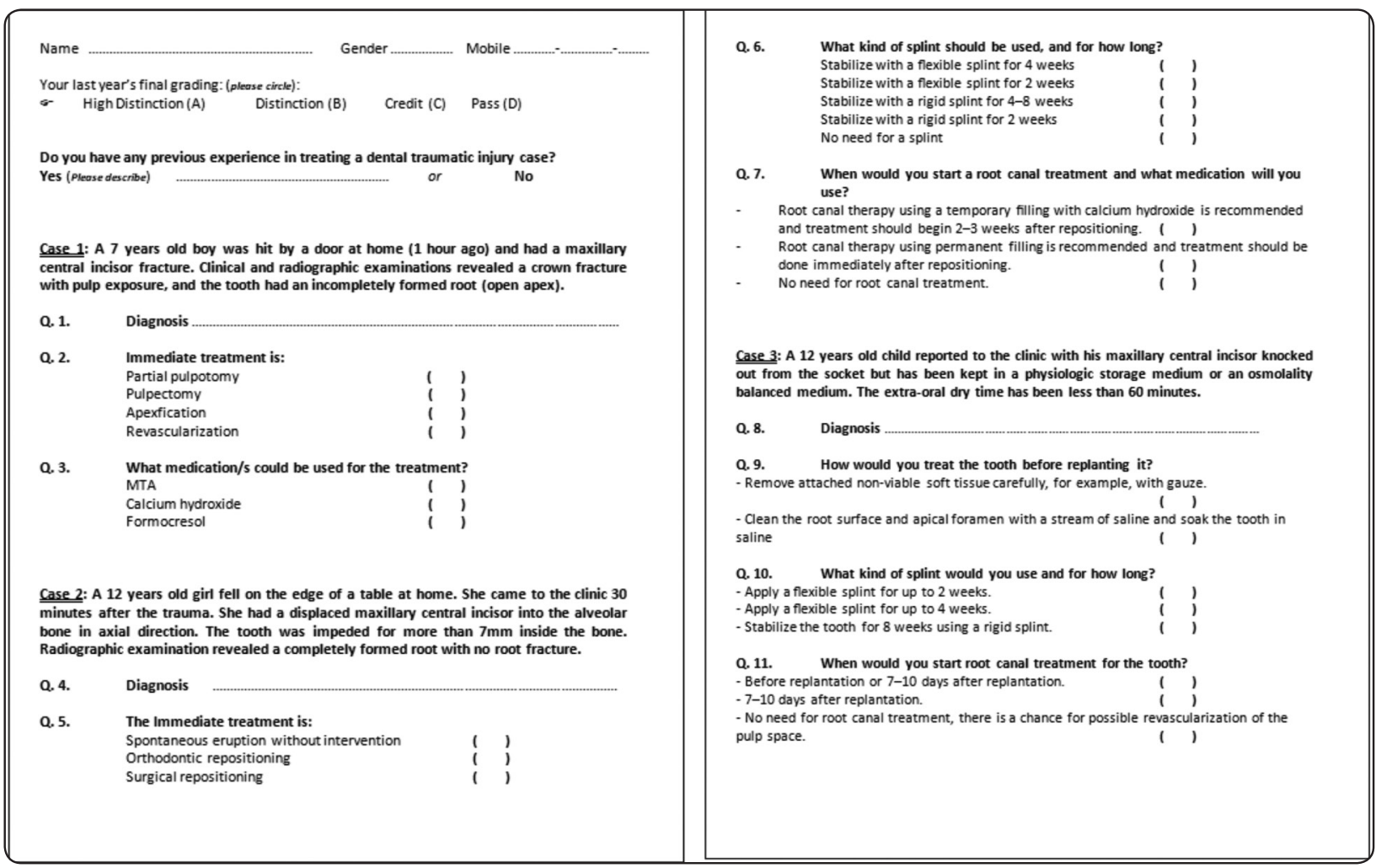

Fig. (1) The Questionnaire for evaluating students' knowledge.

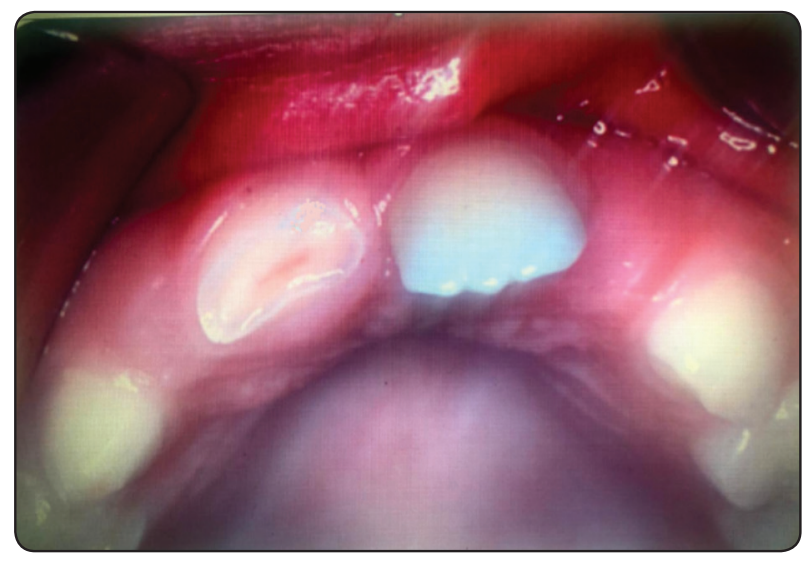

Fig. (2) Complicated crown fracture.

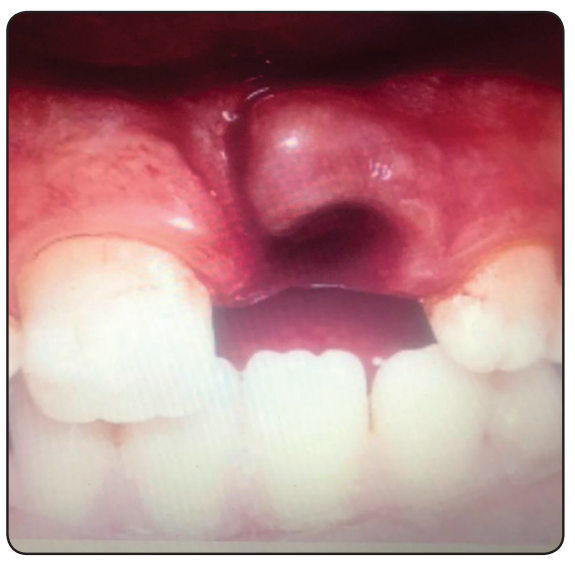

Fig. (3) Intrusive luxation 


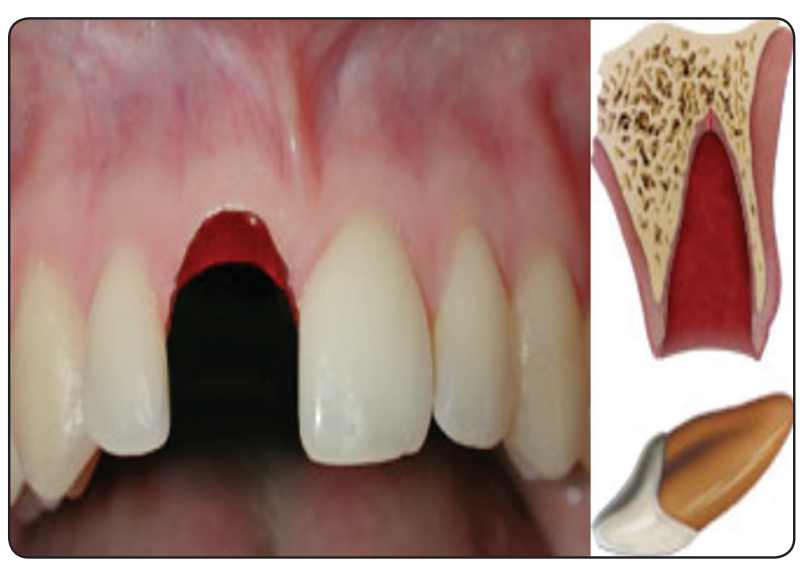

Fig. (4) Avulsion.
Association of Dental Traumatology (IADT). The Application poses specific questions, and presents pictures for various injuries. Once a selection is made: the Application displays a diagnosis based on analysis of answers and selections made by the user $^{(16)}$ (Fig. 5, 6).

The effect of using the Application on student's ability in reaching a diagnosis and in proposing treatment plans for the different traumatic injuries was then statistically analyzed.

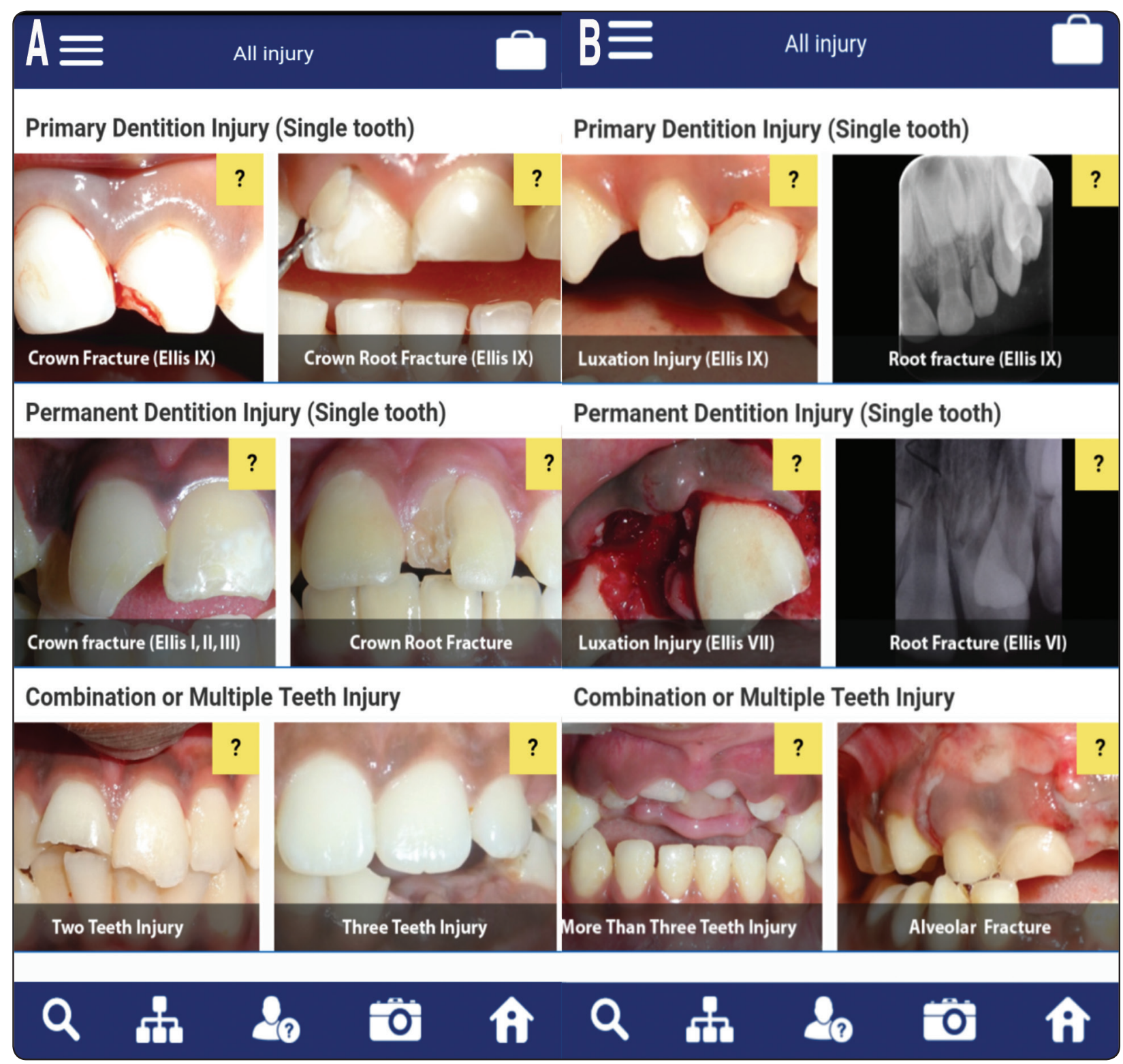




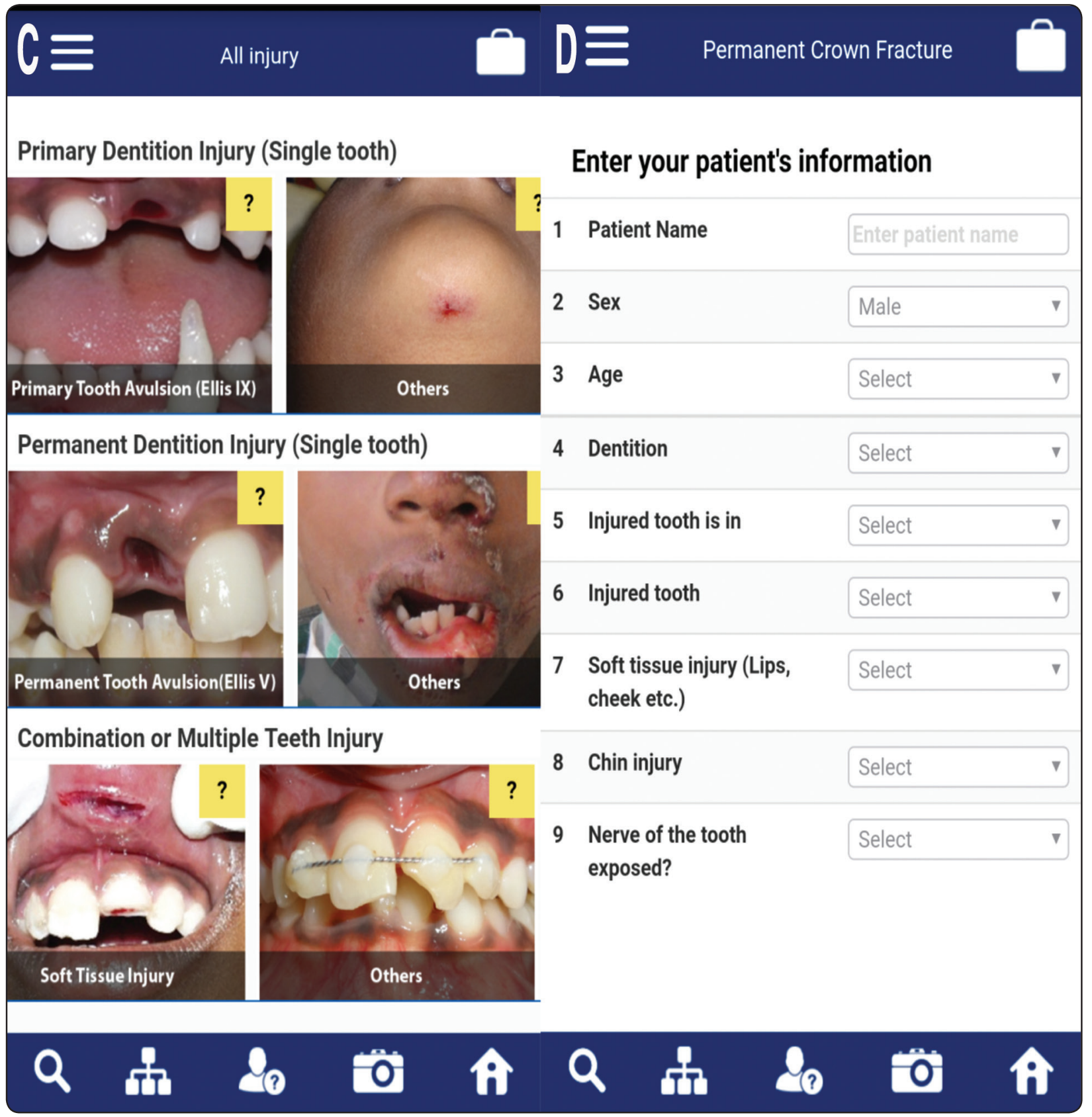

Fig. (5) (a, b, c, d) 'Injured Tooth' Screenshots for different traumatic injuries and data fields' entry. 


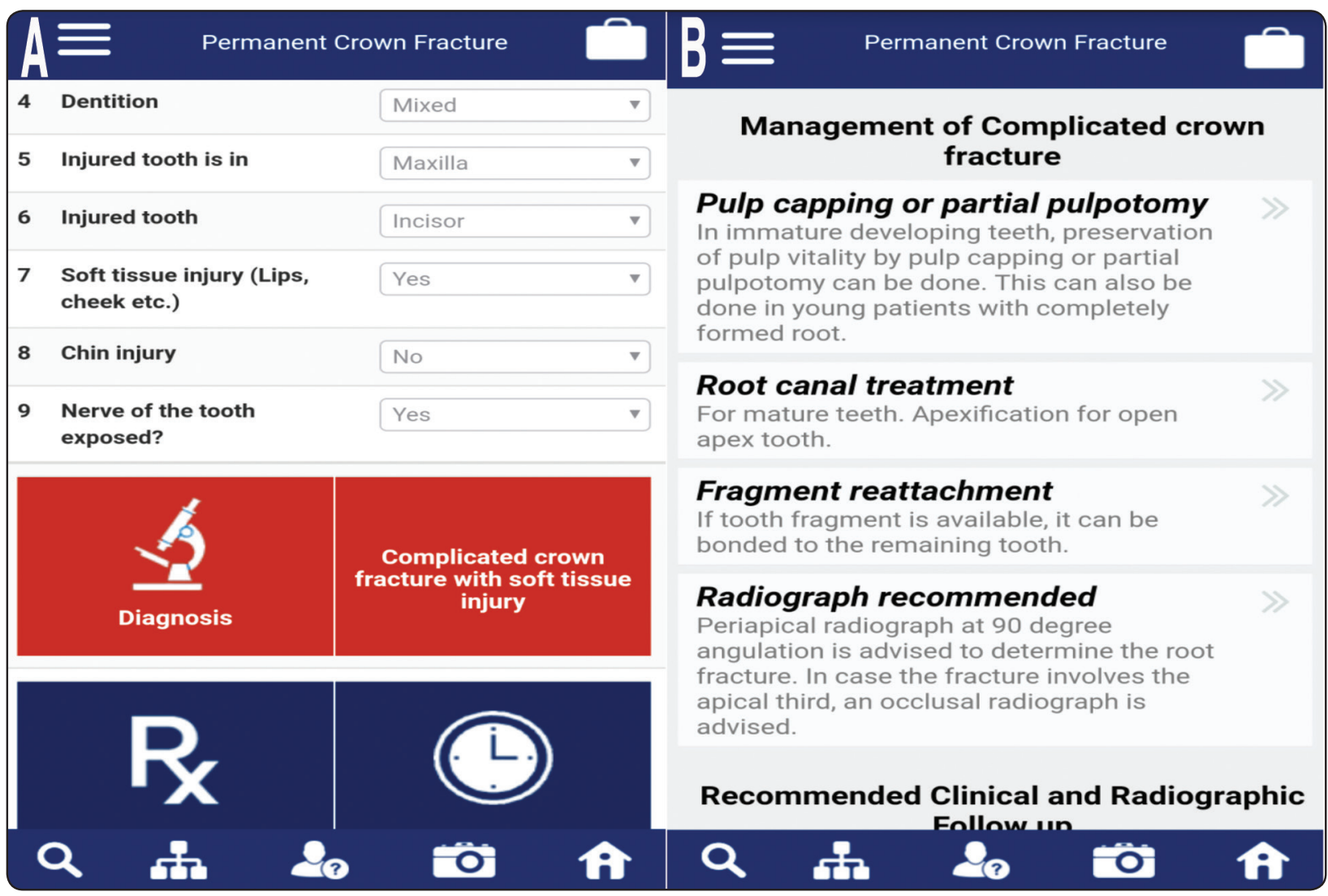

Fig. (6) ‘Injured Tooth' Screen shots for various diagnosis (a) and potential managements (b).

\section{Statistical Analysis:}

Numerical data were presented as mean, standard deviation (SD), median and range values. Qualitative data were presented as frequencies and percentages. McNemar's test was used to compare between percentage of correct answers before and after using the application. Wilcoxon signed-rank test was used to compare between questionnaire scores before and after using the application. Linear regression model was used to determine significant predictors of questionnaire scores. The significance level was set at $P \leq 0.05$. Statistical analysis was performed with IBM SPSS Statistics for Windows, Version 23.0. Armonk, NY: IBM Corp.

\section{RESULTS:}

\section{Demographic data:}

The present study was conducted on 274 dental students; 92 males (33.6\%) and 182 females (66.4\%). Fifty students (18.2\%) had Grade A in the previous year, 113 students (41.2\%) had Grade B, 80 students (29.2\%) had Grade C and 31 students (11.3\%) had Grade D. Only 36 students (13.1\%) had previous experience in treating dental traumatic injury cases.

\section{Questionnaire answers:}

Frequency and percentage distribution of answers to the questionnaire are presented in 
Table (1). Percentages of the correct answers are presented in table (2). The highest percentage of correct answers (98.5\%) was found with Question 3, what medication/s could be used for the treatment? (calcium hydroxide / MTA), followed by Question 8, Diagnosis of the third case, (Avulsion) which recorded a percentage of $94.5 \%$ then by Question 1, Diagnosis of the first case, (Complicated crown fracture) which recorded a percentage of $93.4 \%$ then Question 4, Diagnosis of the second case, (Intrusive luxation) which recorded a percentage of $92.7 \%$. The least percentage of correct answers $(42.3 \%)$ was found with Question 6 ,Type and duration of splint to be used for the second case, (flexible splint for 4 weeks ) followed by Question 10 ,Type and duration of splint to be used for the third case, (flexible splint for up to 2 weeks) which recorded a percentage of $59.1 \%$. The mean percentage of correct answers for all the questions asked in the questionnaire was $77.8 \%$ (SD 17.3).

Each correct answer was given a score of (1) and the wrong answer a score of (0) giving a total scores of (11). The mean (SD) values for the total score were 8.4 (1.4) with a median of 8 and a range of $5-11$.

TABLE (1) Frequency and percentage distribution of answers in the questionnaire

\begin{tabular}{lc}
\hline \multicolumn{1}{c}{ question } & Number (\%) \\
\hline Case 1: A 7 years old boy was hit by a door & \\
at home (1 hour ago) and had a maxillary & \\
central incisor fracture. Clinical and \\
radiographic examinations revealed a crown \\
fracture with pulp exposure, and the tooth \\
had an incompletely formed root (open \\
apex). \\
\hline Q. 1. Diagnosis(Complicated crown fracture) & $256(93.4 \%)$ \\
\hline Q. 2. Immediate treatment is: & $213(77.7 \%)$ \\
\hline Partial pulpotomy & $9(3.3 \%)$ \\
\hline Pulpectomy & $26(9.5 \%)$ \\
\hline Apexfication
\end{tabular}

\begin{tabular}{lc}
\hline Revascularization & $29(10.6 \%)$ \\
\hline $\begin{array}{l}\text { Q. 3. What medication/s could be used for } \\
\text { the treatment? }\end{array}$ \\
\hline MTA & $64(23.4 \%)$ \\
\hline Calcium hydroxide & $238(86.9 \%)$ \\
\hline Formocresol & $4(1.5 \%)$ \\
\hline
\end{tabular}

Case 2: A 12 years old girl fell on the edge

of a table at home. She came to the clinic

30 minutes after the trauma. She had a

displaced maxillary central incisor into the alveolar bone in axial direction. The tooth was impeded for more than $7 \mathrm{~mm}$ inside the bone. Radiographic examination revealed a completely formed root with no root fracture.

\begin{tabular}{lc}
\hline Q. 4. Diagnosis (Intrusive luxation) & $254(92.7 \%)$ \\
\hline Q. 5. The Immediate treatment is & \\
\hline Spontaneous eruption without intervention & $1(0.4 \%)$ \\
\hline Orthodontic repositioning & $108(39.4 \%)$ \\
\hline Surgical repositioning & $163(59.5 \%)$ \\
\hline
\end{tabular}

Q. 6.What kind of splint should be used, and for how long?

\begin{tabular}{lc}
\hline Stabilize with a flexible splint for 4 weeks & $116(42.3 \%)$ \\
\hline Stabilize with a flexible splint for 2 weeks & $114(41.6 \%)$ \\
\hline Stabilize with a rigid splint for 4-8 weeks & $20(7.3 \%)$ \\
\hline Stabilize with a rigid splint for 2 weeks & $14(5.1 \%)$ \\
\hline No need for a splint & $9(3.3 \%)$ \\
\hline $\begin{array}{l}\text { Q. 7. When would you start a root canal } \\
\text { treatment and what medication will you use? }\end{array}$
\end{tabular}
treatment and what medication will you use?

Root canal therapy using a temporary filling 208 (75.9\%) with calcium hydroxide is recommended and treatment should begin 2-3 weeks after repositioning.

Root canal therapy using permanent filling is $54(19.7 \%)$ recommended and treatment should be done immediately after repositioning.

No need for root canal treatment. $10(3.6 \%)$

Case 3: A 12 years old child reported to the clinic with his maxillary central incisor knocked out from the socket but has been kept in a physiologic storage medium or an osmolality balanced medium. The extra-oral dry time has been less than 60 minutes.

Q. 8. Diagnosis(Avulsion) $259(94.5 \%)$ 


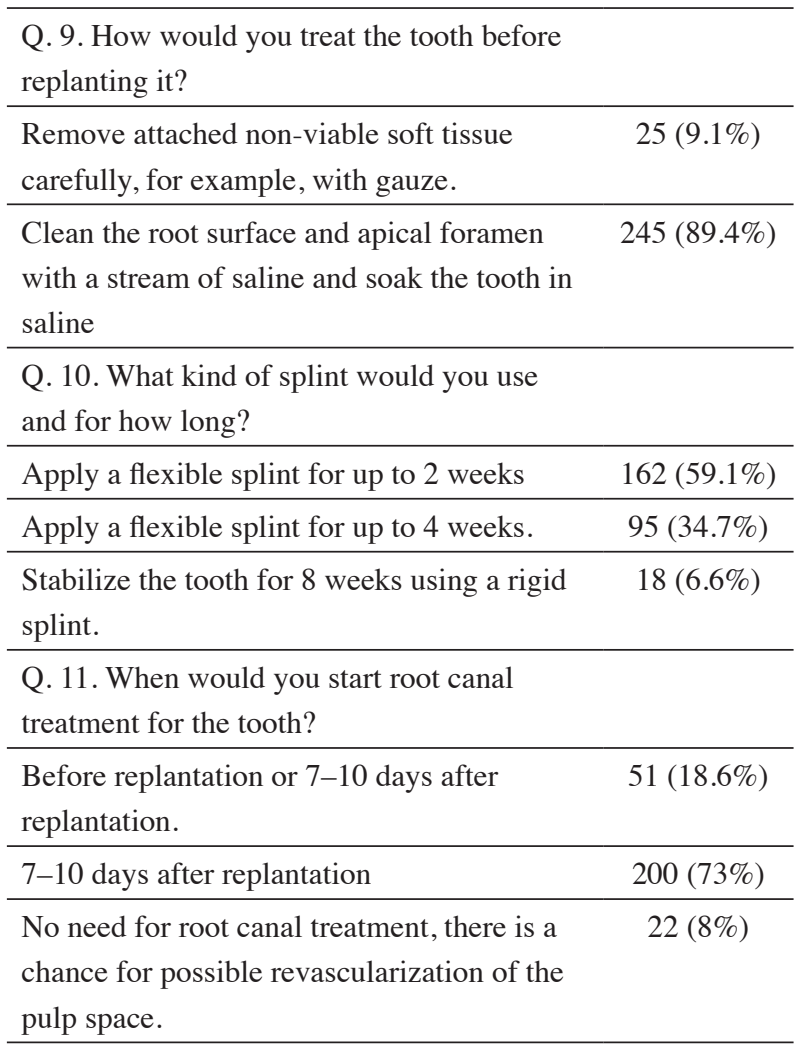

TABLE (2): Frequencies (n) and percentages (\%) of correct answers by all students $(n=274)$

\begin{tabular}{ccc}
\hline Question & $\mathrm{n}$ & $\%$ \\
\hline Q1 & 256 & 93.4 \\
\hline Q2 & 213 & 77.7 \\
\hline Q3 & 270 & 98.5 \\
\hline Q4 & 254 & 92.7 \\
\hline Q5 & 163 & 59.5 \\
\hline Q6 & 116 & 42.3 \\
\hline Q7 & 208 & 75.9 \\
\hline Q8 & 259 & 94.5 \\
\hline Q9 & 245 & 89.4 \\
\hline Q10 & 162 & 59.1 \\
\hline Q11 & 200 & 73 \\
\hline
\end{tabular}

TABLE (3): Frequencies (n), percentages (\%) and results of McNemar's test for comparison between correct answers before and after using the application $(n=58)$

\begin{tabular}{lccccc}
\hline & \multicolumn{2}{c}{ Before using the application } & \multicolumn{2}{c}{ After using the application } & \multirow{2}{*}{$P$-value } \\
\cline { 2 - 5 } Question & $\mathrm{n}$ & $\%$ & $\mathrm{n}$ & $\%$ & 1.000 \\
\hline Q1 & 57 & 98.3 & 58 & 100 & $<0.001^{*}$ \\
Q2 & 40 & 69 & 58 & 100 & $\mathrm{NC}^{\dagger}$ \\
Q3 & 58 & 100 & 58 & 100 & $<0.001^{*}$ \\
Q4 & 46 & 58 & 100 & $<0.001^{*}$ \\
Q5 & 79.3 & 53 & 91.4 & $<0.001^{*}$ \\
Q6 & 44.8 & 44 & 75.9 & $<0.001^{*}$ \\
Q7 & 34.5 & 58 & 100 & $0.004^{*}$ \\
Q8 & 30 & 51.7 & 58 & 100 & $<0.001^{*}$ \\
Q9 & 49 & 54.5 & 58 & 100 & $<0.001^{*}$ \\
Q10 & 45 & 77.6 & 54 & 93.1 & $<0.001^{*}$ \\
Q11 & 36 & 62.1 & 54 & 93.1 & \\
\hline
\end{tabular}




\section{Effect of using the application:}

Students who had wrong answers in the Questionnaire were clustered into three categories: those who got wrong diagnoses, those who got wrong answers, and those whose selected answers were considered to have deleterious effect on treatment and prognosis. Sixty students were selected from those three categories, and asked to proceed to Phase B of the Study to study the effect of using the application on the improvement of answers. Only 58 were available to complete the second stage. They comprised 26 males (44.8\%) and 32 females (55.2\%). Four students (6.9\%) had Grade A, 28 students (48.3\%) had Grade B, 17 students (29.3\%) had Grade $\mathrm{C}$ and 9 students (15.5\%) had Grade D. Only 6 students $(10.3 \%)$ had previous experience in treating dental traumatic injury cases.

Percentages of correct answers before and after using the application are presented in Table (3). There is a statistically significant increase in prevalence of correct answers regarding all questions. Comparison between median scores before and after using the application is presented in Figure (7). The mean (SD) values for the total scores before using the application were $7.6(1.2)$ with a median of 8 and a range of $5-10$ which increased after using the application to a mean (SD) values of $10.5(0.8)$ with

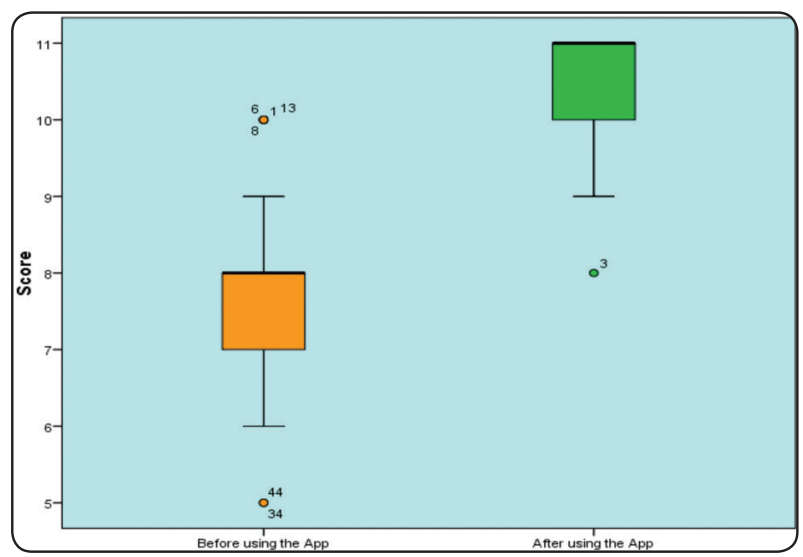

Fig. (7) Box plot representing median and range values for the total score before and after using the application (Circles represent outliers) a median of 11 and a range of $8-11$. This increase is statistically significant as indicated by Wilcoxon signed rank test $(P$-value $<0.001$, Effect size $=$ $0.785)$. The mean percentage of correct answers of the students without the applications is 68.97 (SD 20.2). The mean percentage of correct answers of the students using the application is 95.8 (SD 7.0).

\section{Significant predictors of questionnaire scores:}

Linear regression analysis model was constructed to determine significant predictors of scores. The dependent variable was questionnaire scores while the independent variables were gender, last year grade and previous experience in treating dental trauma cases.

The results showed that gender was the only statistically significant predictor of scores. Females showed statistically significantly higher mean score [8.6 (1.4)] than males [7.9 (1.4)].

TABLE (4): Results of linear regression analysis for the significant predictors of questionnaire scores.

\begin{tabular}{|l|c|c|c|c|c|}
\hline \multirow{2}{*}{ Variables } & \multirow{2}{*}{$\mathrm{B}$} & \multirow{2}{*}{$\mathrm{SE}$} & \multicolumn{2}{|c|}{$95 \% \mathrm{CI}$} & \multirow{2}{*}{ P-value } \\
\cline { 4 - 5 } & & & $\begin{array}{c}\text { Lower } \\
\text { limit }\end{array}$ & $\begin{array}{c}\text { Upper } \\
\text { limit }\end{array}$ & \\
\hline Gender & -0.703 & 0.181 & -1.058 & -0.347 & $<0.001 *$ \\
\hline
\end{tabular}

$B$ : Regression coefficient, SE: Standard error, *: Significant at $P \leq 0.05$

\section{DISCUSSION}

\section{Students' knowledge about dental trauma :}

In regard to traumatic dental injuries, undergraduate teaching should aim in providing the students with adequate knowledge to be able to give a correct diagnosis, propose a correct treatment plan and to prevent wrong treatment that could endanger the case prognosis ${ }^{(7)}$. 
Students' knowledge of dental traumatology is based on the organization of university classes. This part of the study evaluated the theoretical knowledge of dental traumatology of the final year students using a questionnaire including three different scenarios of dental traumatic injuries.

Questions regarding the diagnosis of the three cases had the highest scores of the correct answers. Correct diagnosis of the third case (Avulsion) scored 94.5\% followed by first case (complicated crown fracture) $93.4 \%$ then the second case (intrusive luxation) $92.7 \%$.

Complicated crown fracture is considered more common traumatic dental injury for the students to diagnose, however the difference in the mother language resulted in few cases of wrong diagnosis due to mixing the nomenclature, writing the diagnosis as "complete crown fracture".

According to the International Association of Dental Traumatology guidelines for the management of traumatic dental injuries ${ }^{(4)}$, partial pulpotomy is indicated for complicated crown fracture in a tooth with incomplete root formation. $77.7 \%$ of the students got the correct answer. $20 \%$ of the students selected apexfication or revascularization as the treatment of choice they explained later that their choice based on the time elapsed sense the fracture. They mistakenly considered 60 minute is too long for the pulp to survive.

High score of correct answers of question no. 3 regarding the medication used for partial pulpotomy treatment of the first case is due to the selection of the most popular pulp capping material known to the students which is calcium hydroxide. Pulp capping with calcium hydroxide is a familiar treatment in the practice of the students using similar procedures for caries or traumatic exposures of the pulp ${ }^{(17)}$.

Surgical repositioning of intruded tooth with more than $7 \mathrm{~mm}$ intrusion is recommended by the dental trauma guidelines. Despite of having the highest score of student selection (59.5\%), among the three choices of question 5 (spontaneous eruption without intervention, orthodontic repositioning or surgical repositioning), a considerable percentage of the students (39.4\%) preferred the less aggressive approach of orthodontic repositioning paying no attention to the depth of the intrusion.

Splinting of traumatized teeth was a matter of conflict among the students. Type of the splint, rigid or flexible, duration of application, 2 weeks or 4 weeks is a matter of debate in the literatures. The (IADT) recommended the use of short term flexible splint for replanted tooth with duration not more than 2 weeks and for 4 weeks for the intruded tooth. In question 6 flexible splinting was the common selection of the students for splinting intruded tooth however, selection of the duration was nearly equally distributed between 2 weeks (41.6\%) and four weeks $(42.3 \%)$.

Answers of question 10 revealed the same agreement of most of students on using flexible splint however, the correct selected duration was 2 weeks $(59.1 \%$ ), while $34.7 \%$ selected a splinting duration of 4 weeks which is unnecessary as the periodontal healing occurs in one week ${ }^{(18)}$.

Root canal treatment of the intruded tooth with complete root formation is recommended as the pulp will likely become necrotic in such tooth ${ }^{4}$. Using an intermediate filling as calcium hydroxide is recommended and the treatment should start after 2-3 weeks of repositioning the intruded tooth. $75.9 \%$ of the students got the correct answer, while only $3.6 \%$ thought that root canal treatment is not needed.

An avulsed permanent incisor with completely formed root which is kept in a physiologic storage medium and replanted in time less than 60 minutes has the opportunity of regaining a healthy periodontal attachment but root canal treatment is mandatory. Students who selected the correct answer for the treatment protocol of such tooth before replantation 
were $245(89.4 \%) .25$ students $(9.1 \%)$ choose to remove the attached periodontal tissues with gauze before replanting it. When we discussed their selection after the end of the study they justified their selection being based on information from literatures that the maximum extra oral dry time for favorable prognosis of an avulsed tooth is less than 30 minutes ${ }^{(19)}$. They actually missed that the tooth presented in the questionnaire was kept in a physiologic storage medium.

The recommended time of starting root canal treatment for a replanted tooth with completely formed root is 7-10 days after replantation (4). Pulpal necrosis will occur after an avulsion injury. If endodontic treatment is not carried out, the pulp space will inevitably become infected. The combination of microbes in the root canal and cemental damage on the external surface of the root results in an external inflammatory resorption that can be very aggressive. Resorption will continue as long as the microbes are not removed from the root canal and can lead to the rapid loss of the tooth ${ }^{(19)} .73 \%$ of the students choose the correct answer while $8 \%$ thought that root canal treatment is not needed and that there is a chance for possible revascularization of the pulp space. Most of those students along with those who choose to remove the attached periodontal tissues with gauze before replanting the avulsed tooth were included in the selected students to proceed to the second phase of the study using the smart phone application to answer the questionnaire.

The mean percentage of the correct answers of all the questions in the questionnaire at the first phase was 77.8 (SD 17.3) this result is comparable with the results of Alzoubiet al ${ }^{(7)}$ who found the percentage after giving a single lecture on dental trauma is 83.19 (SD 15.0) and to the results of Karapinar et al ${ }^{(8)}$ where the average percentage of the students' correct answers in their study was 73.33(SD 16.91). The mean value of the total score out of 11 questions was 8.4 (SD1.4) with a median of $8 / 11$ and range of $5-11$. This is comparable to the average of Fujita et al $64.79 / 6$ however; their questionnaire was restricted to avulsed case only. The mean score of the current study is better than that of the study done by Mohebbi et al ${ }^{(9)}$ (5.48 out of 12 questions).

The independent variables were gender, last year grade and previous experience in treating dental trauma cases. The results showed that gender was the only statistically significant predictor of scores. Females showed statistically significantly higher mean score 8.6 (1.4) than males 7.9 (1.4). This result came in accordance to the finding of Alzoubi et al ${ }^{(7)}$ where males scored less marks than females but with no statistical significance. Also Re et al ${ }^{(17)}$ found that the level of knowledge of women was generally better than male colleagues the difference was statistically significant. On the other hand gender was found to have no effect on the score of the correct answers in other studies ${ }^{(8,9,20)}$.

\section{Effect of using the smartphone dental application:}

The use of mobile technology for educational purposes is known as mobile learning ( $\mathrm{m}$ - learning). Faria et al (21) reviewed 215 smartphone dental applications, 38 (17.67\%) of them for Teaching and learning purposes. Regarding the teaching and learning process, the authors highlighted the importance of using smartphone applications as a new tool available to facilitate learning. Teaching and learning methods should be dynamic and in line with social needs and demands. The current generation of students was born and grew up surrounded by and involved with information technology, which permeates their social relations and in general their perception of the world ${ }^{(22)}$. Information and communication technology has proved to be a critical component of teaching and learning in higher education and in the education of health professionals. Mobile devices can contribute 
significantly to a new way of educating in health ${ }^{(23)}$.

The second phase of the study was to evaluate the effect of using Injured Tooth application on the level of knowledge of the final year dental students. Students for the second phase were selected from those who got wrong diagnoses, those who got high score of wrong answers, and those whose selected answers were considered to have deleterious effect on treatment and prognosis. Sixty students were selected from those three categories, and asked to proceed to Phase B. Only 58 were available to complete the second stage.

Using the application, there was a total improvement in the diagnosis of the 3 cases proposed in the questionnaire. An improvement from $93.3 \%$ (complicated crown fracture), $79.3 \%$ (intrusive luxation) and $84.5 \%$ (avulsion) to $100 \%$ in the diagnosis of the 3 cases was significant and as a result, it supports the finding of Mohan et al (16) where they find that the percentages of correct diagnosis given by the application in regard to the 3 cases presented in our questionnaire were $98 \%$, $83.3 \%$ and $100 \%$ respectively.

Improvement of question 2 answers with the increase of the correct answers percentage from $69 \%$ to $100 \%$ is due to the clear instruction in the application at the first glance to the management of complicated crown fracture in immature developing teeth.

Correct answers of Question 5 regarding the method of repositioning an intruded tooth with a depth more than $7 \mathrm{~mm}$ was improved from $44.8 \%$ to $91.8 \%$. The answer of the question is presented in detail in the treatment plan proposed by the application which is based on the dental trauma guidelines however the application is limited in the data entry of the case to the displacement of the tooth into the bone, while the different situation of the intruded tooth wither with complete root or not and the depth of the intrusion is not included in the data entry asked from the application user. This may be the cause of confusion of some students during searching for the correct answer through the different alternatives listed by the application.

This reason could be applied also for the improvement of the answers of question 6 where the percentage of the correct answers increased from 34.5 to 75.9 .

Improvement in the percentage of the correct answers in question 7 was significantly high with increase from 51.7 to 100 . This is because this is the only treatment protocol for root canal treatment proposed in the application for the intruded tooth, so students had no confusion by other alternatives as in the other areas of the treatment plan.

A very satisfactory result were obtained from using the application in regard to question 9 , as $100 \%$ of the students selected the right answer regarding how to manage the avulsed tooth before replantation. This may be due to the presences of all the possibilities in the data entry regarding the avulsed case and the direct recommended treatment plan that the user get after entering all the needed data.

This is also applied to the level of improvement in the correct answers' percentage of both questions no 10 and 11.

The use of the application was effective in providing accessible knowledge to the students to guide them in proposing the possible management protocols for the different traumatic dental injuries presented in this study. This conclusion is in agreement with the conclusion of a similar study done by Al-Musawi et al ${ }^{(24)}$ where they used a smartphone application, Dental Trauma App, as an intervention in comparison to a 30-min lecture on the emergency management of tooth avulsion to assess its use effect on the knowledge of emergency management in a group of school teachers. They concluded that The Dental Trauma App alone is an effective means of providing accessible knowledge to guide laypeople in managing tooth avulsion, and it can be superior to a lecture-based delivery of information. 


\section{CONCLUSIONS}

1- Knowledge of the students regarding traumatic dental injuries is efficient in some areas while considered low in other parts.

2- The use of the application was effective in providing accessible knowledge to the students to guide them in proposing the possible management protocols for the different traumatic dental injuries presented in this study.

\section{REFERENCES}

1- Petti, S., Glendor, U., and Andersson, L. World traumatic dental injury prevalence and incidence, a meta-analysis One billion living people have had traumatic dental injuries. Dental Traumatology 2018;34, 71-86.

2- Shalan H. and Abo Bakr R. "Oral Health Status of Preschool Children in Egypt". Acta Scientific Dental Sciences 2018; 67-72.

3- Awad M. and Hegazy S. Prevalence and risk factors of traumatic dental injuries to permanent anterior teeth among 8-12 years old school children in Egypt. Pediatric Dental Journal 2016; 26(2): 67-73.

4- Andreasen JO, Lauridsen E, Gerds TA, Ahrensburg SS. Dental Trauma Guide: a source of evidencebased treatment guidelines for dental trauma. Dent Traumatology 2012; 28: 345-350.

5- Treating traumatic dental injuries. Updated Guidelines from the AAE. J Calif Dent Assoc. 2014; 42: 14.

6- Fujita Y, Shiono Y, Maki K. Knowledge of emergency management of avulsed tooth among Japanese dental students. BMC Oral Health [Internet]. 2014;14(1):1-6. Available from: BMC Oral Health

7- Alzoubi F, Mannocci F, Newton T, Manoharan A, Djemal S. What do dental students know about trauma? Dent Traumatol. 2015;31(6):482-6.

8- Karapinar-Kazandag M, Tanalp J, Ayhan T, Kaptan RF, Ersev H. Evaluation of retention of dental students' trauma knowledge following a reminder lecture. Biomed Res. 2018;29(9):1756-63.

9- Mohebbi SZ, Razeghi S. Dental students' knowledge about emergency management of dental trauma. J Craniomax Res. 2017; 4(1):296-300).
10- Soubra BN, Debs NN. Impact of audiovisual method in educating children facing dental avulsion. Dent Traumatology 2014; 30: 216-221.

11- Ho,A. and Quick, O. 'Leaving patients to their own devices? Smart technology, safety and therapeutic relationships', BMC Medical Ethics. BMC Medical Ethics, 2018; 19(1), pp. 1-6. doi: 10.1186/s12910-018-0255-8

12- Buijink AWG, Visser BJ, Marshall L. Medical apps for smartphones: lack of evidence undermines quality and safety. Evid Based Med 2013; 18: 90 -92.

13- Djemal S, Singh P. Smartphones and dental trauma: the current availability of apps for managing traumatic dental injuries. Dent Traumatology 2016; 32: 52 -57.

14- https://play.google.com/store/apps/details?id=com . iadtapp.toothsos\&hl=en_US.Accessed on 20 March 2019.

15- https://play.google.com/store/apps/details?id=com.abh. injuredtooth\&hl=en_USAccessed on 20 March 2019.

16- Mohan A, Agarwal T, Cherian T et al. Diagnostic ability of a smart phone app (injured tooth) in diagnosing traumatic injuries to the teeth - a multicentre analysis. International Journal of Paediatric Dentistry 2018; 28: 561-569.

17- Re D, Augusti D, Paglia G, Augusti G, Cotti E. Treatment of traumatic dental injuries: Evaluation of knowledge among Italian dentists. Eur J Paediatr Dent. 2014;15(1):23-8.

18- Kahler B, Heithersay GS. An evidence-based appraisal of splinting luxated, avulsed and root-fractured teeth. Dent Traumatol 2008;24, 2-10.

19- Trope M. Avulsion of permanent teeth: Theory to practice. Dent Traumatol. 2011;27(4):281-94.

20- Hatem M, Taher EM. Libyan general dentists' knowledge of dental trauma management. Int Dent Med J Adv Res Vol 2015. 2017;1(1):1-6.

21- Faria IM, Sancas MC, Pintor AVB, Primo LSG. Dental Apps for Smartphones: New Way of Providing Services and Education. Creat Educ. 2018;09(05):687-96.

22- Schulz P, Sagheb K, Affeldt H, et al. Acceptance of e-learning devices by dental students. Med 2 0. 2013; 2(2):e6. Published 2013 Aug 14. doi:10.2196/med20.2767

23- Deshpande, S., Chahande, J., \& Rathi, A. Mobile Learning App: A Novel Method to Teach Clinical Decision Making in Prosthodontics. Education for Health,2017; 30, 31-34.

24- Al-Musawi A, Al-Sane M, Andersson L. Smartphone App as an aid in the emergency management of avulsed teeth. Dent Traumatol. 2017;33(1):13-8. 